\title{
Striped structures of stable and unstable sets of expansive homeomorphisms and a theorem of K. Kuratowski on independent sets
}

\author{
by
}

\author{
Hisao Ka to (Hiroshima)
}

\begin{abstract}
We investigate striped structures of stable and unstable sets of expansive homeomorphisms and continuum-wise expansive homeomorphisms. The following theorem is proved: if $f: X \rightarrow X$ is an expansive homeomorphism of a compact metric space $X$ with $\operatorname{dim} X>0$, then the decompositions $\left\{W^{\mathrm{s}}(x) \mid x \in X\right\}$ and $\left\{W^{\mathrm{u}}(x) \mid x \in X\right\}$ of $X$ into stable and unstable sets of $f$ respectively are uncountable, and moreover there is $\sigma$ $(=\mathrm{s}$ or $\mathrm{u})$ and $\varrho>0$ such that there is a Cantor set $C$ in $X$ with the property that for each $x \in C, W^{\sigma}(x)$ contains a nondegenerate subcontinuum $A_{x}$ containing $x$ with $\operatorname{diam} A_{x} \geq \varrho$, and if $x, y \in C$ and $x \neq y$, then $W^{\sigma}(x) \neq W^{\sigma}(y)$. For a continuum-wise expansive homeomorphism, a similar result is obtained. Also, we prove that if $f: G \rightarrow G$ is a map of a graph $G$ and the shift map $\tilde{f}:(G, f) \rightarrow(G, f)$ of $f$ is expansive, then for each $\widetilde{x} \in(G, f), W^{\mathrm{u}}(\widetilde{x})$ is equal to the arc component of $(G, f)$ containing $\widetilde{x}$, and $\operatorname{dim} W^{\mathrm{s}}(\widetilde{x})=0$.
\end{abstract}

1. Introduction. All spaces under consideration are assumed to be metric. By a compactum we mean a compact metric space, and by a continuum a connected nondegenerate compactum. A homeomorphism $f: X \rightarrow X$ of a compactum $X$ is called expansive [6] if there is a constant $c>0$ (called an expansive constant for $f$ ) such that if $x, y \in X$ and $x \neq y$, then there is an integer $n=n(x, y) \in \mathbb{Z}$ such that

$$
d\left(f^{n}(x), f^{n}(y)\right)>c .
$$

This property has frequent applications in topological dynamics, ergodic theory and continuum theory $[1,5,6,25]$.

A homeomorphism $f: X \rightarrow X$ of a compactum $X$ is continuum-wise expansive [15] if there is a constant $c>0$ such that if $A$ is a nondegener-

1991 Mathematics Subject Classification: Primary 54B25, 54F50; Secondary 54H20, 54E40, 54B20.

Key words and phrases: expansive homeomorphism, continuum-wise expansive homeomorphism, stable and unstable sets, independent. 
ate subcontinuum of $X$, then there is an integer $n=n(A) \in \mathbb{Z}$ such that $\operatorname{diam} f^{n}(A)>c$. By the definitions, we can easily see that every expansive homeomorphism is continuum-wise expansive, but the converse is not true. There are many important examples of homeomorphisms which are continuum-wise expansive, but not expansive.

In [13, Theorem 3.1], we proved that if $f: X \rightarrow X$ is an expansive homeomorphism of a compactum $X$ with $\operatorname{dim} X>0$, then there is a closed subset $Z$ of $X$ such that each component of $Z$ is nondegenerate, the space of components of $Z$ is a Cantor set, the decomposition space of $Z$ into components is upper and lower semi-continuous and all components of $Z$ are contained in stable sets or unstable sets.

In this paper, we show more precise results. In particular, we prove that if $f: X \rightarrow X$ is an expansive homeomorphism of a compactum $X$ with $\operatorname{dim} X>0$, then the decompositions $\left\{W^{\mathrm{s}}(x) \mid x \in X\right\}$ and $\left\{W^{\mathrm{u}}(x) \mid x\right.$ $\in X\}$ of $X$ into stable and unstable sets respectively are uncountable, and moreover there is $\sigma(\sigma=\mathrm{s}$ or $\mathrm{u})$ and $\varrho>0$ such that the $\sigma$-striped set $Z(\sigma, \varrho)$ of $f$ is not empty. Hence, by using a theorem of K. Kuratowski on independent sets, it is proved that almost every Cantor set $C$ of $Z(\sigma, \varrho)$ has the property that for each $x \in C, W^{\sigma}(x)$ contains a nondegenerate subcontinuum containing $x$ and if $x, y \in C$ and $x \neq y$, then $W^{\sigma}(x) \neq W^{\sigma}(y)$.

Also, we prove that if $f: G \rightarrow G$ is a map of a graph $G$ and the shift map $\widetilde{f}:(G, f) \rightarrow(G, f)$ of $f$ is expansive, then for each $\widetilde{x} \in(G, f), W^{\mathrm{u}}(\widetilde{x})$ is the arc component of $(G, f)$ containing $\widetilde{x}$, and $W^{\mathrm{s}}(\widetilde{x})$ is 0 -dimensional.

We refer the readers to [1], [6] and [25] for the general properties of expansive homeomorphisms.

2. Definitions and preliminaries. Let $X$ be a metric space. Then the hyperspaces $2^{X}$ and $C(X)$ of $X$ are defined as follows:

$$
\begin{gathered}
2^{X}=\{A \mid A \text { is a nonempty compact subset of } X\}, \\
C(X)=\left\{A \in 2^{X} \mid A \text { is connected }\right\} .
\end{gathered}
$$

The hyperspaces $2^{X}$ and $C(X)$ are metric spaces with the Hausdorff metric $d_{\mathrm{H}}$, i.e., $d_{\mathrm{H}}(A, B)=\inf \left\{\varepsilon>0 \mid U_{\varepsilon}(A) \supset B\right.$ and $\left.U_{\varepsilon}(B) \supset A\right\}$, where $U_{\varepsilon}(A)$ denotes the $\varepsilon$-neighborhood of $A$ in $X$. Note that if $X$ is a compactum, then the hyperspaces $2^{X}$ and $C(X)$ are also compacta (see [21]). Let $A$ and $B$ be subsets of $X$. Put $d(A, B)=\inf \{d(a, b) \mid a \in A$ and $b \in B\}$.

Let $f: X \rightarrow X$ be a homeomorphism of a compactum $X$ and let $x \in X$. Then the stable set $W^{\mathrm{s}}(x)$ and the unstable set $W^{\mathrm{u}}(x)$ are defined as follows:

$$
\begin{aligned}
& W^{\mathrm{s}}(x)=\left\{y \in X \mid \lim _{n \rightarrow \infty} d\left(f^{n}(x), f^{n}(y)\right)=0\right\}, \\
& W^{\mathrm{u}}(x)=\left\{y \in X \mid \lim _{n \rightarrow \infty} d\left(f^{-n}(x), f^{-n}(y)\right)=0\right\} .
\end{aligned}
$$


Also, the continuum-wise stable and unstable sets $V^{\mathrm{s}}(x), V^{\mathrm{u}}(x)$ are defined as follows:

$$
\begin{aligned}
& V^{\mathrm{s}}(x)=\{y \in X \mid \text { there is } A \in C(X) \text { such that } x, y \in A \text { and } \\
& \left.\qquad \lim _{n \rightarrow \infty} \operatorname{diam} f^{n}(A)=0\right\}, \\
& V^{\mathrm{u}}(x)=\{y \in X \mid \text { there is } A \in C(X) \text { such that } x, y \in A \text { and } \\
& \left.\lim _{n \rightarrow \infty} \operatorname{diam} f^{-n}(A)=0\right\} .
\end{aligned}
$$

Clearly, $W^{\sigma}(x) \supset V^{\sigma}(x),\left\{W^{\sigma}(x) \mid x \in X\right\}$ and $\left\{V^{\sigma}(x) \mid x \in X\right\}$ are decompositions of $X$ for both $\sigma=$ s and u, i.e., $X=\bigcup\left\{W^{\sigma}(x) \mid x \in X\right\}$ (resp. $X=\bigcup\left\{V^{\sigma}(x) \mid x \in X\right\}$ ), and if $W^{\sigma}(x) \neq W^{\sigma}(y)$ (resp. $V^{\sigma}(x) \neq$ $V^{\sigma}(y)$ ), then $W^{\sigma}(x) \cap W^{\sigma}(y)=\emptyset$ (resp. $\left.V^{\sigma}(x) \cap V^{\sigma}(y)=\emptyset\right)$. Also, for $0<\delta<\varepsilon$ consider the following subsets of $C(X)$ :

$$
\begin{aligned}
V^{\mathrm{s}}(\varepsilon) & =\left\{A \in C(X) \mid \operatorname{diam} f^{n}(A) \leq \varepsilon \text { for any } n \geq 0\right\}, \\
V^{\mathrm{u}}(\varepsilon) & =\left\{A \in C(X) \mid \operatorname{diam} f^{-n}(A) \leq \varepsilon \text { for any } n \geq 0\right\}, \\
V^{\mathrm{s}}(\delta, \varepsilon) & =\left\{A \in V^{\mathrm{s}}(\varepsilon) \mid \operatorname{diam} A=\delta\right\}, \\
V^{\mathrm{u}}(\delta, \varepsilon) & =\left\{A \in V^{\mathrm{u}}(\varepsilon) \mid \operatorname{diam} A=\delta\right\}, \\
V^{\mathrm{s}} & =\left\{A \in C(X) \mid \lim _{n \rightarrow \infty} \operatorname{diam} f^{n}(A)=0\right\}, \\
V^{\mathrm{u}} & =\left\{A \in C(X) \mid \lim _{n \rightarrow \infty} \operatorname{diam} f^{-n}(A)=0\right\} .
\end{aligned}
$$

We are interested in the structures of the decompositions $\left\{W^{\sigma}(x) \mid x \in\right.$ $X\}$ and $\left\{V^{\sigma}(x) \mid x \in X\right\}(\sigma=\mathrm{s}$ and $\mathrm{u})$ of $X$. Let $f: X \rightarrow X$ be an expansive homeomorphism of a compactum $X$ with an expansive constant $c>0$ and $\operatorname{dim} X>0$. Let $c>\varrho>0$ be a positive number. Consider the family $\Phi(\sigma)=\{Z \mid Z$ is a closed subset of $X$ such that (i) for each $x \in Z$ there is a subcontinuum $A_{x}$ of $X$ with $\operatorname{diam} A_{x} \geq \varrho$ and $x \in A_{x} \subset W^{\sigma}(x)$, and (ii) for any neighborhood $U$ of $x$ in $X$, there is $y \in Z \cap U$ such that $\left.W^{\sigma}(x) \neq W^{\sigma}(y)\right\}$. By [20, p. 315], $\Phi(\sigma)$ has the maximal element $Z(\sigma, \varrho)$ $(=\operatorname{Cl}(\bigcup\{Z \mid Z \in \Phi(\sigma)\}))$. The set $Z(\sigma, \varrho)$ is said to be a $\sigma$-striped set of $f$. Note that if $0<\varrho_{1}<\varrho_{2}$, then $Z\left(\sigma, \varrho_{1}\right) \supset Z\left(\sigma, \varrho_{2}\right)$. Also, note that if $Z(\sigma, \varrho) \neq \emptyset$ for some $\varrho>0$, then $X$ contains an uncountable collection of mutually disjoint, nondegenerate subcontinua of $X$ each of which is contained in a different element of $\left\{W^{\sigma}(x) \mid x \in X\right\}$ (see (3.1)).

Let $f: X \rightarrow X$ be a map of a compactum $X$ with metric $d$. Consider the following inverse limit space:

$$
(X, f)=\left\{\left(x_{i}\right)_{i=0}^{\infty} \mid x_{i} \in X, f\left(x_{i+1}\right)=x_{i} \text { for each } i \geq 0\right\} .
$$

Define a metric $\widetilde{d}$ for $(G, f)$ by

$$
\widetilde{d}(\widetilde{x}, \widetilde{y})=\sum_{i=0}^{\infty} d\left(x_{i}, y_{i}\right) / 2^{i} \quad \text { for } \widetilde{x}=\left(x_{i}\right)_{i=0}^{\infty}, \widetilde{y}=\left(y_{i}\right)_{i=0}^{\infty} \in(X, f) .
$$


The space $(X, f)$ is called the inverse limit of the map $f$. Define a map $\tilde{f}:(X, f) \rightarrow(X, f)$ by

$$
\widetilde{f}\left(x_{0}, x_{1}, \ldots\right)=\left(f\left(x_{0}\right), x_{0}, x_{1}, \ldots\right) \quad \text { for }\left(x_{i}\right)_{i=0}^{\infty} \in(X, f) .
$$

Then $\tilde{f}$ is a homeomorphism and it is called the shift map of $f$. Let $p_{n}$ : $(X, f) \rightarrow X$ be the natural projection $(n \geq 0)$, i.e., $p_{n}\left(\left(x_{i}\right)_{i=0}^{\infty}\right)=x_{n}$.

(2.1) ExAmple. Let $f: I \rightarrow I$ be the homeomorphism as in Figure 1, where $I=[0,1]$ denotes the unit interval.

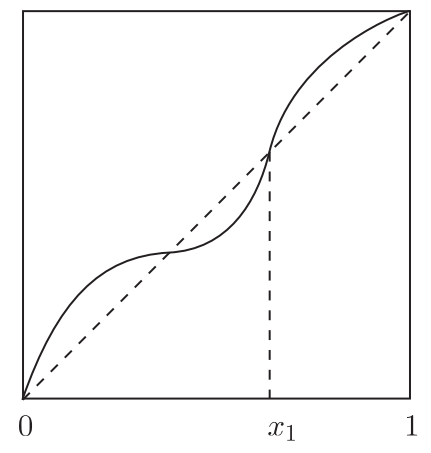

Fig. 1

Then $\left\{W^{\mathrm{s}}(x) \mid x \in I\right\}=\left\{\{0\},\left(0, x_{1}\right),\left\{x_{1}\right\},\left(x_{1}, 1\right]\right\}$ is finite, because $W^{\mathrm{s}}(0)=\{0\}, W^{\mathrm{s}}(y)=\left(0, x_{1}\right)$ for $y \in\left(0, x_{1}\right), W^{\mathrm{s}}\left(x_{1}\right)=\left\{x_{1}\right\}$ and $W^{\mathrm{s}}(x)=$ $\left(x_{1}, 1\right]$ for $x \in\left(x_{1}, 1\right]$. Similarly, $\left\{W^{\mathrm{u}}(x) \mid x \in I\right\}$ is finite. Hence $Z(\sigma, \varrho)=\emptyset$ for each $\varrho>0(\sigma=\mathrm{s}$ and $\mathrm{u})$.

(2.2) ExAmple. Let $S^{1}$ be the unit circle and let $f: S^{1} \rightarrow S^{1}$ be the natural covering map with degree 2. Consider the inverse limit $\left(S^{1}, f\right)$ of $f$ and the shift map $\tilde{f}:\left(S^{1}, f\right) \rightarrow\left(S^{1}, f\right)$. The continuum $\left(S^{1}, f\right)$ is well-known as the 2-adic solenoid and $\widetilde{f}$ is an expansive homeomorphism (see [26]). In this case, for each $\widetilde{x} \in\left(S^{1}, f\right), W^{\mathrm{u}}(\widetilde{x})=V^{\mathrm{u}}(\widetilde{x})$ is the arc component of $\left(S^{1}, f\right)$ containing $\widetilde{x}$. Also, $V^{\mathrm{s}}(\widetilde{x})=\{\widetilde{x}\} \varsubsetneqq W^{\mathrm{s}}(\widetilde{x})$ for each $\widetilde{x} \in\left(S^{1}, f\right)$. Then the decomposition $\left\{W^{\sigma}(\widetilde{x}) \mid \widetilde{x} \in\left(S^{1}, f\right)\right\}(\sigma=\mathrm{s}$ and $\mathrm{u})$ is uncountable. Note that $\operatorname{dim} W^{\mathrm{s}}(\widetilde{x})=0$, because $W^{\mathrm{s}}(\widetilde{x})$ is an $F_{\sigma}$-set and does not contain a nondegenerate subcontinuum (see (3.10) below). Note that the continuum $\left(S^{1}, f\right)$ itself is a u-striped set $Z(\mathrm{u}, \varrho)$ of $\widetilde{f}$ for some $\varrho>0$, but $Z(\mathrm{~s}, \varrho)=\emptyset$ for each $\varrho>0$.

(2.3) ExAmple. (a) There is an expansive homeomorphism $f: X \rightarrow X$ such that $\operatorname{Int}_{X} W^{\sigma}(x) \neq \emptyset$ for some $x \in X$. Let $G$ be the one-point union of the unit interval $I$ and a circle $S^{1}$, i.e., $(G, *)=(I, 1) \vee\left(S^{1}, *\right)$. Define a map $g: G \rightarrow G$ such that $g \mid S^{1}: S^{1} \rightarrow S^{1}$ is the natural covering map with degree 2 and $g(0)=0, g(1)=*$ and $g(I)=G$. We can choose $g: G \rightarrow G$ 
so that $\widetilde{g}: X=(G, g) \rightarrow X=(G, g)$ is expansive (see [10, Theorem 4.3]). Then $W^{\mathrm{u}}(\widetilde{0})$ is a dense open set of $X$, where $\widetilde{0}=(0,0, \ldots)$. Hence $X$ itself is not a u-striped set of $\widetilde{g}$.

(b) There is an expansive homeomorphism $h$ of a continuum $Y$ such that there is a point $x_{0} \in Y$ such that if $A$ is any nondegenerate subcontinuum of $Y$ containing $x_{0}$, then $A \notin V^{\mathrm{s}} \cup V^{\mathrm{u}}$. Let $G$ and $g: G \rightarrow G$ be the same as in (a), and let $X_{i}(i=1,2)$ be the copies of the space $X$ as in (a). Let $(Y, \widetilde{0})=\left(X_{1}, \widetilde{0}\right) \vee\left(X_{2}, \widetilde{0}\right)$ be the one-point union of $X_{1}$ and $X_{2}$ (see Figure 2).

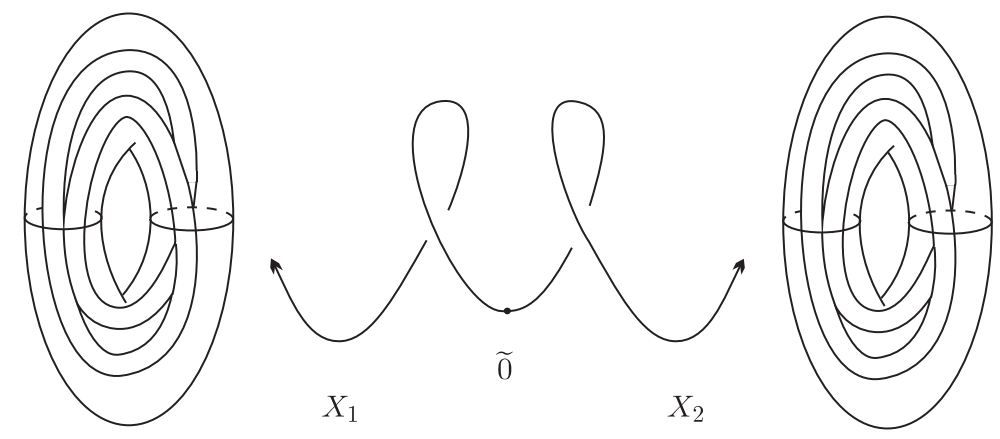

Fig. 2

Take a natural injection $i: \mathbb{R} \rightarrow Y$ such that $i(0)=\widetilde{0}$, where $\mathbb{R}$ is the set of real numbers. Let $k: \mathbb{R} \rightarrow \mathbb{R}$ be defined by $k(x)=2 x+1(x \geq 0)$, $k(x)=\frac{1}{2} x+1(x \leq 0)$. Define a homeomorphism $h: Y \rightarrow Y$ by $h(x)=\widetilde{g}(x)$ if $x \in\left(S^{1}, g \mid S^{1}\right) \subset X_{1}, h(x)=\widetilde{g}^{-1}(x)$ if $x \in\left(S^{1}, g \mid S^{1}\right) \subset X_{2}$ and $h(x)=$ $i \circ k \circ i^{-1}(x)$ if $x \in i(\mathbb{R})$. Then $h$ is an expansive homeomorphism. Note that if $x_{0} \in i(\mathbb{R})$ and $A$ is any nondegenerate subcontinuum containing $x_{0}$, then $A \notin V^{\mathrm{s}} \cup V^{\mathrm{u}}$.

Remark. Instead of the solenoid $\left(S^{1}, g\right)$, one can construct the examples above with the help of an Anosov diffeomorphism, say $\left(\begin{array}{ll}2 & 1 \\ 1 & 1\end{array}\right)$ on the 2-dimensional torus $T^{2}$, and a curve outside $T^{2}$ "unwinding" from an unstable manifold in $T^{2}$.

A subset $E$ of a space $X$ is an $F_{\sigma^{-}}$set in $X$ if $E$ is the union of a countable collection of closed subsets of $X$. A subset $E$ of $X$ is an $F_{\sigma \delta^{-}}$set in $X$ if it is the intersection of a countable collection of $F_{\sigma}$-sets.

In this paper, we use a theorem of K. Kuratowski on independent sets [19]. A subset $F$ of $X$ is said to be independent in $R \subset X^{n}$ if for every system $x_{1}, \ldots, x_{n}$ of different points of $F$ the point $\left(x_{1}, \ldots, x_{n}\right) \in F^{n}$ never belongs to $R$. In [19], K. Kuratowski proved the following theorem.

(2.4) Theorem ([19, Main theorem and Corollary 3$])$. If $X$ is a complete space and $R \subset X^{n}$ is an $F_{\sigma}$-set of the first category, then the set $J(R)$ of 
all compact subsets $F$ of $X$ independent in $R$ is a dense $G_{\delta}$-set in the space $2^{X}$ of all compact subsets of $X$. Moreover, if $X$ has no isolated points, then almost every Cantor set of $X$ is independent in $R$.

(2.5) Proposition. Let $f: X \rightarrow X$ be a homeomorphism of a compactum $X$. Then $W^{\sigma}(x)$ is an $F_{\sigma \delta}$-set in $X(\sigma=\mathrm{s}, \mathrm{u})$.

Proof. We prove the case $\sigma=\mathrm{u}$. Let $x \in X$. For any natural numbers $m, n \geq 1$, consider the set

$$
W_{m, n}(x)=\left\{y \in X \mid d\left(f^{-i}(x), f^{-i}(y)\right) \leq 1 / n \text { for } i \geq m\right\} .
$$

Then $W_{m, n}(x)$ is closed and $W_{n}(x)=\bigcup_{m=1}^{\infty} W_{m, n}(x)$ is an $F_{\sigma}$-set. Hence $W^{\mathrm{u}}(x)=\bigcap_{n=1}^{\infty} W_{n}(x)$ is an $F_{\sigma \delta}$-set in $X$.

(2.6) Proposition. Let $f: X \rightarrow X$ be an expansive homeomorphism of a compactum $X$. Then $W^{\sigma}(x)$ is an $F_{\sigma}$-set in $X(\sigma=\mathrm{s}, \mathrm{u})$.

Proof. We prove the case $\sigma=u$. Let $c>0$ be an expansive constant for $f$ and let $0<\varepsilon<c / 2$. Note that if $y, y^{\prime} \in X$ and $d\left(f^{-i}(y), f^{-i}\left(y^{\prime}\right)\right) \leq \varepsilon$ for each $i \geq m$ ( $m$ is some natural number), then $\lim _{i \rightarrow \infty} d\left(f^{-i}(y), f^{-i}\left(y^{\prime}\right)\right)=0$ (see $[20$, p. 315$]$ ). For any $m=1,2, \ldots$, put

$$
W_{m, \varepsilon}(x)=\left\{y \in X \mid d\left(f^{-i}(x), f^{-i}(y)\right) \leq \varepsilon \text { for } i \geq m\right\} .
$$

Since $W^{\mathrm{u}}(x)=\bigcup_{m=1}^{\infty} W_{m, \varepsilon}(x)$ and $W_{m, \varepsilon}(x)$ is closed, $W^{\mathrm{u}}(x)$ is an $F_{\sigma}$-set in $X$.

(2.7) Proposition. Let $f: X \rightarrow X$ be a continuum-wise expansive homeomorphism of a compactum $X$. Then $V^{\sigma}(x)$ is an $F_{\sigma}$-set in $X(\sigma=$ $\mathrm{s}, \mathrm{u})$.

Proof. We prove the case $\sigma=\mathrm{u}$. Let $c>0$ be a continuum-wise expansive constant for $f$ and let $0<\varepsilon<c / 2$. Note that if $A \in C(X)$ and $\operatorname{diam} f^{-i}(A) \leq \varepsilon$ for any $i \geq m$, then $\lim _{i \rightarrow \infty} \operatorname{diam} f^{-i}(A)=0$ (see $[15$, $(2.1)])$. For each $m=1,2, \ldots$, put

$$
V_{m, \varepsilon}(x)=\bigcup\left\{A \in C(X) \mid x \in A \text { and } \operatorname{diam} f^{-i}(A) \leq \varepsilon \text { for } i \geq m\right\} .
$$

Then $V^{\mathrm{u}}(x)=\bigcup_{m=1}^{\infty} V_{m, \varepsilon}(x)$ is an $F_{\sigma}$-set in $X$.

3. Striped structures of stable and unstable sets. In this section, we study striped structures of stable and unstable sets of expansive homeomorphisms and continuum-wise expansive homeomorphisms. The main result of this section is the following theorem.

(3.1) Theorem. Let $f: X \rightarrow X$ be an expansive homeomorphism of a compactum $X$ with $\operatorname{dim} X>0$. Then the decomposition $\left\{W^{\sigma}(x) \mid x \in X\right\}$ $(\sigma=\mathrm{s}$ and $\mathrm{u})$ of $X$ is uncountable. Moreover, there exists $\sigma(\sigma=\mathrm{s}$ or $\mathrm{u})$ and $\varrho>0$ such that the $\sigma$-striped set $Z(\sigma, \varrho)$ is not empty. In particular, almost 
every Cantor set $C$ of $Z(\sigma, \varrho)$ has the property that for any $x \in C$, there exists a nondegenerate subcontinuum $A_{x}$ of $X$ such that $x \in A_{x} \subset W^{\sigma}(x)$, and if $x, y \in C$ and $x \neq y$, then $W^{\sigma}(x) \neq W^{\sigma}(y)$.

To prove (3.1), we need the following facts. The next lemma is obvious.

(3.2) Lemma. Let $f: X \rightarrow X$ be a map of a compactum $X$ and let $N \geq 1$ be a natural number. Suppose that there is $\gamma>0$ such that $d\left(f^{i N}(x), f^{i N}(y)\right)$ $\geq \gamma$ for each $i=0,1,2, \ldots$ Then there is $\eta>0$ such that $d\left(f^{i}(x), f^{i}(y)\right) \geq \eta$ for each $i=0,1,2, \ldots$

(3.3) Lemma ([15, (2.3)]). Let $f: X \rightarrow X$ be a continuum-wise expansive homeomorphism of a compactum $X$ with an expansive constant $c>0$ and let $0<\varepsilon<c / 2$. Then there is $\delta>0$ such that if $A$ is any nondegenerate subcontinuum of $X$ such that $\operatorname{diam} A \leq \delta$ and $\operatorname{diam} f^{m}(A) \geq \varepsilon$ for some integer $m \in \mathbb{Z}$, then one of the following conditions holds:

(a) If $m \geq 0$, then $\operatorname{diam} f^{n}(A) \geq \delta$ for each $n \geq m$. More precisely, there is a subcontinuum $B$ of $A$ such that $\operatorname{diam} f^{j}(B) \leq \varepsilon$ for $0 \leq j \leq n$ and $\operatorname{diam} f^{n}(B)=\delta$.

(b) If $m<0$, then $\operatorname{diam} f^{-n}(A) \geq \delta$ for each $n \geq-m$. More precisely, there is a subcontinuum $B$ of $A$ such that $\operatorname{diam} f^{-j}(B) \leq \varepsilon$ for $0 \leq j \leq n$ and $\operatorname{diam} f^{-n}(B)=\delta$.

(3.4) Lemma $([15,(2.4)])$. Let $f, c, \varepsilon, \delta$ be as in (3.3). Then for any $\gamma>0$, there is $N>0$ such that if $A \in C(X)$ and $\operatorname{diam} A \geq \gamma$, then either $\operatorname{diam} f^{n}(A) \geq \delta$ for each $n \geq N$, or $\operatorname{diam} f^{-n}(A) \geq \delta$ for each $n \geq N$.

Pro of of (3.1). Let $c, \varepsilon, \delta$ be positive numbers as in (3.3). Suppose that there exists no nondegenerate subcontinuum $A$ of $X$ such that $\lim _{n \rightarrow \infty} \operatorname{diam} f^{-n}(A)=0$, i.e., $V^{\mathrm{u}}=\{\{x\} \mid x \in X\}$. Let $C$ be a nondegenerate component of $X$. Then for any $x \in C, W^{\mathrm{u}}(x)=\bigcup_{i=1}^{\infty} F_{i}$, where each $F_{i}$ is closed (see (2.6)). Note that $\operatorname{Int}_{C}\left(F_{i} \cap C\right)=\emptyset$ for all $i$. By the Baire category theorem, $\left\{W^{\mathrm{u}}(x) \mid x \in C\right\}$ is uncountable, and hence $\left\{W^{\mathrm{u}}(x) \mid x \in X\right\}$ is uncountable. The case $\sigma=\mathrm{s}$ is similar.

Next, we shall show the existence of a nonempty $\sigma$-striped set $Z(\sigma, \varrho)$. By [20, Lemma 3], there is a nondegenerate subcontinuum $A \in V^{\mathrm{s}} \cup V^{\mathrm{u}}$. From now on, we assume that there is a nondegenerate subcontinuum $A$ such that $\lim _{i \rightarrow \infty} \operatorname{diam} f^{-i}(A)=0$. In this case, $V^{\mathrm{u}}(\delta, \varepsilon) \neq \emptyset$ (see $\left.(3.3)\right)$. Note that if $A \in V^{\mathrm{u}}$, then $W^{\mathrm{u}}(x)=W^{\mathrm{u}}(y)$ for all $x, y \in A$.

For any closed subset $M$ of $V^{\mathrm{u}}(\varepsilon)$, we define

$$
\begin{aligned}
M^{f}=\{A \in C(X) \mid & \text { for any neighborhood } \mathbf{U} \text { of } A \text { in } C(X) \text { there is } \\
& A^{\prime} \in M \text { such that } A^{\prime} \in \mathbf{U} \text { and } W^{\mathrm{u}}(a) \cap W^{\mathrm{u}}\left(a^{\prime}\right)=\emptyset \\
& \text { for all } \left.a \in A \text { and } a^{\prime} \in A^{\prime}\right\} .
\end{aligned}
$$


We can easily see that $M^{f} \subset M$ is a closed subset of $C(X)$ and $\left(M^{f}\right)^{f}$ $\subset M^{f}$. For any ordinal numbers, define $M_{0}=M, M_{1}=M^{f}, M_{\alpha+1}=$ $\left(M_{\alpha}\right)^{f}$, and $M_{\lambda}=\bigcap_{\alpha<\lambda} M_{\alpha}$, where $\lambda$ is a limit ordinal. From now on, we assume that

$$
M=M(\delta)=V^{\mathrm{u}}(\delta, \varepsilon) .
$$

Now, we need the following claim which is directly proved by transfinite induction.

Claim $(\lambda)$. Let $\lambda$ be a countable ordinal. If $A \in M_{\lambda}$, then there are two subcontinua $A_{1}$ and $A_{2}$ of $A$ such that $d\left(A_{1}, A_{2}\right) \geq \delta / 3$ and $A_{1}, A_{2} \in\left(M^{\prime}\right)_{\lambda}$, where $M^{\prime}=M(\gamma)\left(=V^{\mathrm{u}}(\gamma, \varepsilon)\right)$ and $0<\gamma<\delta / 3$.

By transfinite induction, we shall prove $M_{\lambda} \neq \emptyset$ for any ordinal $\lambda$. Choose $\gamma>0$ such that if $A$ and $B$ are any subsets of $X$ with diam $A \geq \delta$ and $\operatorname{diam} B \geq \delta$, then there are $a \in A$ and $b \in B$ such that $d(a, b) \geq 3 \gamma$. Let $N$ be a natural number such that if $A \in C(X)$ and $\operatorname{diam} A \geq \gamma$, then either $\operatorname{diam} f^{n}(A) \geq \delta(n \geq N)$ or $\operatorname{diam} f^{-n}(A) \geq \delta(n \geq N)$ (see (3.4)). We may assume $\gamma \leq \delta / 3$.

First, choose $A \in V^{\mathrm{u}}(\delta, \varepsilon)$. Since $\operatorname{diam} A=\delta$, we can choose two subcontinua $A_{1}, B_{1}$ of $A$ such that $A_{1}, B_{1} \in M^{\prime}=V^{\mathrm{u}}(\gamma, \varepsilon)$ and $d\left(A_{1}, B_{1}\right) \geq$ $\delta / 3 \geq \gamma$ (see Claim (0)). Since $\operatorname{diam} f^{N}\left(A_{1}\right) \geq \delta$ and $\operatorname{diam} f^{N}\left(B_{1}\right) \geq \delta$, we choose a subcontinuum $A_{2}$ of $f^{N}\left(A_{1}\right)$ and a subcontinuum $B_{2}$ of $f^{N}\left(B_{1}\right)$ such that $A_{2}, B_{2} \in V^{\mathrm{u}}(\gamma, \varepsilon)$ and $d\left(A_{2}, B_{2}\right) \geq \gamma$. By induction, we can choose two sequences $\left\{A_{n}\right\}$ and $\left\{B_{n}\right\}$ of $C(X)$ such that $A_{n} \subset f^{N}\left(A_{n-1}\right)$, $B_{n} \subset f^{N}\left(B_{n-1}\right), d\left(A_{n}, B_{n}\right) \geq \gamma$ and $A_{n}, B_{n} \in V^{\mathrm{u}}(\gamma, \varepsilon)$. Also, choose a subsequence $n_{1}<n_{2}<\ldots$ of natural numbers such that $\lim _{i \rightarrow \infty} A_{n_{i}}=A^{\prime}$ and $\lim _{i \rightarrow \infty} B_{n_{i}}=B^{\prime}$. Then $d\left(f^{-N i}\left(A^{\prime}\right), f^{-N i}\left(B^{\prime}\right)\right) \geq \gamma$ for each $i \geq 0$. By (3.2), $d\left(f^{-i}(a), f^{-i}(b)\right) \geq \eta$ for all $a \in A^{\prime}, b \in B^{\prime}$ and $i \geq 0$, and hence $W^{\mathrm{u}}(a) \neq W^{\mathrm{u}}(b)$. Note that for each $a_{n_{i}} \in A_{n_{i}}$ and $b_{n_{i}} \in B_{n_{i}}, W^{\mathrm{u}}\left(a_{n_{i}}\right)=$ $W^{\mathrm{u}}\left(b_{n_{i}}\right)$. Hence either $A^{\prime} \in\left(M^{\prime}\right)^{f}=\left(M^{\prime}\right)_{1}$ or $B^{\prime} \in\left(M^{\prime}\right)^{f}=\left(M^{\prime}\right)_{1}$. We assume that $A^{\prime} \in\left(M^{\prime}\right)_{1}$. By $(3.3), f^{N}\left(A^{\prime}\right)$ contains a subcontinuum $A_{1}$ such that $A_{1} \in M_{1}$, which implies that $M_{1} \neq \emptyset$.

For a countable ordinal $\lambda$, we may assume that for any $\alpha<\lambda, M_{\alpha}$ is not empty. We must consider the following two cases.

(I) $\lambda=\alpha+1$. Claim $(\alpha)$ and an argument similar to the above show that $M_{\lambda}$ is not empty.

(II) $\lambda$ is a limit ordinal. In this case, take an increasing sequence $\alpha_{1}<$ $\alpha_{2}<\ldots$ of countable ordinals such that $\lim _{i \rightarrow \infty} \alpha_{i}=\lambda$. Also, choose $A_{i} \in M_{\alpha_{i}}$ for each $i$. We may assume that $\lim _{i \rightarrow \infty} A_{i}=A_{\infty}$. Then $A_{\infty} \in$ $\bigcap_{\alpha<\lambda} M_{\alpha}=M_{\lambda}$.

Thus we proved that $M_{\lambda} \neq \emptyset$ for any countable ordinal $\lambda$. Hence there is a countable ordinal $\alpha$ such that $M_{\alpha}=M_{\alpha+1}(\neq \emptyset)$. Put $Z=\bigcup\left\{A \mid A \in M_{\alpha}\right\}$. 
Since $M_{\alpha}$ is closed in $C(X), Z$ is also closed in $X$. We can easily see that $Z=Z(\mathrm{u}, \delta)$ is a $\mathrm{u}$-striped set of $f$. Put

$$
A(n, \varepsilon)=\left\{(x, y) \in Z \times Z \mid d\left(f^{-i}(x), f^{-i}(y)\right) \leq \varepsilon \text { for each } i \geq n\right\} .
$$

Then $A(n, \varepsilon)$ is a closed subset of $Z \times Z$; put $R=\bigcup_{n=1}^{\infty} A(n, \varepsilon)$. Note that $\operatorname{Int}_{Z} A(n, \varepsilon)=\emptyset$. Hence $R$ is an $F_{\sigma}$-set of the first category in $Z \times Z$. By the theorem of K. Kuratowski on independent sets (see (2.4)), $\mathbf{S}=\left\{S \in 2^{Z}\right.$ | $S$ is independent in $R\}=\left\{S \in 2^{Z} \mid\right.$ for any $x, y \in S$ with $x \neq y, W^{\mathrm{u}}(x) \neq$

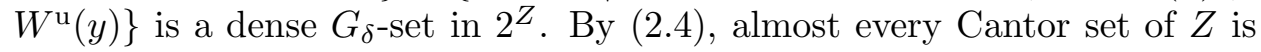
contained in $\mathbf{S}$. This completes the proof.

(3.5) COROllary. Under the assumption of (3.1), if moreover $V^{\mathrm{s}}$ and $V^{\mathrm{u}}$ contain nondegenerate subcontinua, then for both $\sigma=\mathrm{s}$ and $\sigma=\mathrm{u}$, the $\sigma$-striped set $Z(\sigma, \varrho)$ of $f$ is not empty for some $\varrho>0$.

By (2.7) and an argument similar to the above, we can prove the following theorem on continuum-wise expansive homeomorphisms.

(3.6) Theorem. Let $f: X \rightarrow X$ be a continuum-wise expansive homeomorphism of a compactum $X$ with $\operatorname{dim} X>0$. Then the decompositions $\left\{V^{\sigma}(x) \mid x \in X\right\}(\sigma=\mathrm{s}$ and $\mathrm{u})$ are uncountable. Moreover, there is $\sigma$ ( $\sigma=$ $\mathrm{s}$ or $\mathrm{u})$ and a positive number $\varrho>0$ such that there is a nonempty closed set $Z^{\prime}$ of $X$ such that (i) for each $x \in Z^{\prime}$ there is a subcontinuum $A_{x}$ of $X$ with $\operatorname{diam} A_{x} \geq \varrho$ and $x \in A_{x} \subset V^{\sigma}(x)$, (ii) for any neighborhood $U$ of $x$ in $X$, there is $y \in Z^{\prime} \cap U$ such that $V^{\sigma}(x) \neq V^{\sigma}(y)$. In particular, almost every Cantor set $C$ of $Z(\sigma)$ has the property that for any $x \in C$, there is a nondegenerate subcontinuum $A_{x}$ of $X$ with $x \in A_{x} \subset V^{\sigma}(x)$, and if $x, y \in C$ and $x \neq y$, then $V^{\sigma}(x) \neq V^{\sigma}(y)$.

(3.7) Theorem. Let $X$ be a locally connected continuum (= Peano continuum). If $f: X \rightarrow X$ is an expansive homeomorphism (resp. a continuum-wise expansive homeomorphism) of $X$, then there is an uncountable subset $Z$ of $X$ such that $\mathrm{Cl}(Z)=X$, and

(1) for each $x \in Z$ and $\sigma=\mathrm{s}$ and $\mathrm{u}$, there is a nondegenerate subcontinuum $A_{x} \in V^{\sigma}$ with $x \in A_{x}$ and $\operatorname{diam} A_{x} \geq \delta$ for some $\delta>0$,

(2) if $x, y \in Z$ and $x \neq y$, then $W^{\sigma}(x) \neq W^{\sigma}(y)\left(\right.$ resp. $\left.V^{\sigma}(x) \neq V^{\sigma}(y)\right)$ for both $\sigma=\mathrm{s}$ and $\mathrm{u}$.

To prove (3.7), we need the following.

(3.8) Lemma ([16, (1.6)]). Let $f: X \rightarrow X$ be a continuum-wise expansive homeomorphism of a Peano continuum $X$. Then there is $\delta>0$ such that for each $x \in X$, there are two subcontinua $A_{x}$ and $B_{x}$ such that $x \in A_{x} \cap B_{x}, A_{x}$ $\in V^{\mathrm{s}}, B_{x} \in V^{\mathrm{u}}, \operatorname{diam} A_{x}=\delta$ and $\operatorname{diam} B_{x}=\delta$. In particular, $\operatorname{Int}_{X}\left(W^{\sigma}(x)\right)$ $=\emptyset$ for each $x \in X$ and $\sigma=\mathrm{s}, \mathrm{u}$. 
Pr o of of (3.7). Suppose that $f$ is an expansive homeomorphism. The case of continuum-wise expansive homeomorphism is similarly proved. Let $\mathcal{B}=\left\{U_{i}\right\}_{i=1}^{\infty}$ be a base of $X$. We use the Baire category theorem. By induction, we obtain a countable subset $Z_{\omega}$ of $X$ such that $U_{i} \cap Z_{\omega} \neq \emptyset$ and if $x, y \in Z_{\omega}$, then $W^{\sigma}(x) \neq W^{\sigma}(y)$ for $\sigma=\mathrm{s}$ and $\mathrm{u}$, because $\operatorname{Int}_{X}\left(W^{\sigma}(x)\right)=$ $\emptyset$ and $W^{\sigma}(x)$ is an $F_{\sigma}$-set (see (2.6)). By transfinite induction, for any countable ordinal $\lambda$ we have a countable set $Z_{\lambda}$ such that (1) if $x, y \in Z_{\lambda}$ and $x \neq y$, then $W^{\sigma}(x) \neq W^{\sigma}(y)$ for both $\sigma=\mathrm{s}$ and $\mathrm{u}$, and (2) if $\alpha<\beta$, then $Z_{\alpha} \varsubsetneqq Z_{\beta}$. Put $Z=\bigcup_{\lambda<\omega_{1}} Z_{\lambda}$. Clearly, $Z$ is the desired set.

Let $f: X \rightarrow X$ be a homeomorphism of a compactum $X$. For $\sigma=\mathrm{s}$ and $\mathrm{u}$, let $M_{\sigma}(f)$ be the maximal element of the family $\{Z \mid Z$ is a closed subset of $X$ such that for any $x \in Z$ and any neighborhood $U$ of $x$ in $X$ there is $y \in Z \cap U$ such that $\left.W^{\sigma}(x) \neq W^{\sigma}(y)\right\}$. Clearly, $M_{\sigma}(f)$ is $f$-invariant. It is called the $\sigma$-mixed set of $f$.

By (3.1) and (3.7), we have the following corollary.

(3.9) Corollary. If $f: X \rightarrow X$ is an expansive homeomorphism of a compactum $X$ with $\operatorname{dim} X>0$, then the $\sigma$-mixed set $M_{\sigma}(f)$ is not empty and hence it is a perfect set. Moreover, if $X$ is a Peano continuum, then $M_{\sigma}(f)=X$.

Proof. By (3.1), there is an uncountable subset $H_{\sigma}$ of $X$ such that if $x, y \in H_{\sigma}$ and $x \neq y$, then $W^{\sigma}(x) \neq W^{\sigma}(y)$. Then $\mathrm{Cl}\left(H_{\sigma}\right)$ is a closed and uncountable set, hence it contains a Cantor set $C$. Then $C \subset M_{\sigma}(f)$.

For the case of inverse limits of graphs, we have the following theorem.

(3.10) TheOREM. Let $f: G \rightarrow G$ be a map of a graph $G$ (= finite connected 1-dimensional polyhedron). Suppose that the shift map $\widetilde{f}:(G, f) \rightarrow$ $(G, f)$ is expansive. Then for each $\widetilde{x} \in(G, f)$, (a) $W^{\mathrm{u}}(\widetilde{x})$ is equal to the arc component $A(\widetilde{x})$ of $(G, f)$ containing $\widetilde{x}$, and (b) $W^{\mathrm{s}}(\widetilde{x})$ is 0-dimensional.

To prove (3.10), we need the following notations. Let $A$ be a closed subset of a compactum $X$. A map $f: X \rightarrow X$ is called positively expansive on $A$ if there is $c>0$ such that if $x, y \in A$ and $x \neq y$, then there is a natural number $n \geq 0$ such that $d\left(f^{n}(x), f^{n}(y)\right)>c$. If a map $f: X \rightarrow X$ is positively expansive on the whole space $X$, we say $f$ is positively expansive. Let $\mathcal{A}$ be a finite closed covering of $X$. A map $f: X \rightarrow X$ is positively pseudo-expansive with respect to $\mathcal{A}$ if the following conditions hold:

$\left(\mathrm{P}_{1}\right) f$ is positively expansive on $A$ for each $A \in \mathcal{A}$.

$\left(\mathrm{P}_{2}\right)$ For all $A, B \in \mathcal{A}$ with $A \cap B \neq \emptyset$, either $f$ is positively expansive on $A \cup B$, or there is a natural number $k \geq 1$ such that for any $A^{\prime}, A^{\prime \prime} \in \mathcal{A}$ with $A^{\prime} \cap A^{\prime \prime} \neq \emptyset$, either

$$
f^{k}\left(A^{\prime} \cup A^{\prime \prime}\right) \cap(A-B)=\emptyset \quad \text { or } \quad f^{k}\left(A^{\prime} \cup A^{\prime \prime}\right) \cap(B-A)=\emptyset .
$$


(3.11) Theorem $([13,(2.5)])$. Let $G$ be a graph and let $f: G \rightarrow G$ be an onto map. Then the shift map $\widetilde{f}:(G, f) \rightarrow(G, f)$ is expansive if and only if $f$ is positively pseudo-expansive with respect to $\mathcal{A}$, where $\mathcal{A}=\{e \mid$ e is an edge of some simplicial complex $K$ with $|K|=G\}$.

(3.12) Proposition ([13, (2.9)]). Let $f: G \rightarrow G$ be an onto map of a graph $G$. If the shift map $\widetilde{f}:(G, f) \rightarrow(G, f)$ is expansive, then there is $\alpha>0$ such that if $A$ is a subcontinuum of $(G, f)$ with $\operatorname{diam} A \leq \alpha$, then $A \in V^{\mathrm{u}}$, i.e., $\lim _{n \rightarrow \infty} \operatorname{diam} \widetilde{f}^{-n}(A)=0$.

Proof of (3.10). We may assume that $f: G \rightarrow G$ is an onto map, since so is $f \mid G^{\prime}: G^{\prime} \rightarrow G^{\prime}$, where $G^{\prime}=p_{n}((G, f))$ and $p_{n}:(G, f) \rightarrow G$ is the natural projection.

(a) Let $\widetilde{y}$ be any point of the arc component $A(\widetilde{x})$ of $(G, f)$ containing $\widetilde{x}$. Choose an $\operatorname{arc} A$ from $\widetilde{x}$ to $\widetilde{y}$ in $A(\widetilde{x})$. Choose points $\widetilde{x}=\widetilde{x}_{0}, \widetilde{x}_{1}, \ldots, \widetilde{x}_{m}=\widetilde{y}$ of $A$ such that $\operatorname{diam}\left[\widetilde{x}_{i}, \widetilde{x}_{i+1}\right] \leq \alpha$ for each $i=0,1, \ldots, m-1$, where $\alpha>0$ is as in (3.12) and $\left[\widetilde{x}_{i}, \widetilde{x}_{i+1}\right]$ denotes the arc from $\widetilde{x}_{i}$ to $\widetilde{x}_{i+1}$ in $A$. By (3.12), $\left[\widetilde{x}_{i}, \widetilde{x}_{i+1}\right] \in V^{\mathrm{u}}$ for each $i$, hence $\widetilde{y} \in W^{\mathrm{u}}(\widetilde{x})$. This implies that $A(\widetilde{x}) \subset$ $W^{\mathrm{u}}(\widetilde{x})$.

We show the converse inclusion. Let $\widetilde{y} \in W^{\mathrm{u}}(\widetilde{x})$. Suppose that $\widetilde{x}=$ $\left(x_{i}\right)_{i=0}^{\infty}$ and $\widetilde{y}=\left(y_{i}\right)_{i=0}^{\infty}$. Since $\lim _{i \rightarrow \infty} d\left(x_{i}, y_{i}\right)=0$, there is $m \geq 0$ such that if $n \geq m$, then $x_{n} \in e_{n}$ and $y_{n} \in e_{n}^{\prime}$, where $K$ is a simplicial complex as in (3.11) and $e_{n}, e_{n}^{\prime}$ are edges of $K$ such that $e_{n} \cap e_{n}^{\prime} \neq \emptyset$. Also, we assume that $d\left(x_{n}, y_{n}\right)<\min \left\{d\left(e, e^{\prime}\right) \mid e\right.$ and $e^{\prime}$ are edges of $K$ with $\left.e \cap e^{\prime}=\emptyset\right\}$ for each $n \geq m$.

Since $f: G \rightarrow G$ is positively pseudo-expansive with respect to $\mathcal{A}=\{e \mid e$ is an edge of $K\}$, it is positively expansive on $e_{n} \cup e_{n}^{\prime}$ for each $n \geq m$. We may assume that $f\left(\left[x_{n}, y_{n}\right]\right)$ does not contain a simple closed curve, where $\left[x_{n}, y_{n}\right]$ is the arc in $e_{n} \cup e_{n}^{\prime}$ from $x_{n}$ to $y_{n}$. It follows that $f\left(\left[x_{n+1}, y_{n+1}\right]\right)=\left[x_{n}, y_{n}\right]$ and $f \mid\left[x_{n+1}, y_{n+1}\right]:\left[x_{n+1}, y_{n+1}\right] \rightarrow\left[x_{n}, y_{n}\right]$ is a homeomorphism for each $n \geq m$, because $f \mid\left[x_{n}, y_{n}\right]$ is locally injective and $f\left(\left[x_{n}, y_{n}\right]\right)$ does not contain a simple closed curve. Consider the subset $A=\left\{\left(z_{i}\right)_{i=0}^{\infty} \mid z_{i} \in G, z_{n} \in\right.$ $\left[x_{n}, y_{n}\right]$ for each $n \geq m$ and $f\left(z_{i+1}\right)=z_{i}$ for each $\left.i \geq 0\right\}$ in $(G, f)$. Clearly, $A$ is an $\operatorname{arc}$ from $\widetilde{x}$ to $\widetilde{y}$ in $(G, f)$. Hence $\widetilde{y} \in A(\widetilde{x})$. Note that $V^{\mathrm{u}}(\widetilde{x})=W^{\mathrm{u}}(\widetilde{x})$.

(b) By $(3.12), W^{\mathrm{s}}(\widetilde{x})$ contains no nondegenerate subcontinuum. Since $W^{\mathrm{s}}(\widetilde{x})$ is an $F_{\sigma}$-set in $(G, f), W^{\mathrm{s}}(\widetilde{x})=\bigcup_{i=1}^{\infty} F_{i}$, where each $F_{i}$ is closed. Since $\operatorname{dim} F_{i}=0$ for each $i$, by the sum theorem of dimension theory we see that $\operatorname{dim} W^{\mathrm{s}}(\widetilde{x})=0$.

(3.13) Remark. Of course (3.10) is not true for general expansive homeomorphisms. Consider for example an arbitrary Anosov diffeomorphism. Even in the 1-dimensional case (3.10) is not true. Let $g: G \rightarrow G$ be the map as in (a) of $(2.3)$. Let $Y=((G, g), \widetilde{0}) \vee\left((G, g)^{\prime}, \widetilde{0}^{\prime}\right)$ be the one-point 
union of $((G, g), \widetilde{0})$ and $\left((G, g)^{\prime}, \widetilde{0}^{\prime}\right)$, where $\left((G, g)^{\prime}, \widetilde{0^{\prime}}\right)$ is a copy of $((G, g), \widetilde{0})$. Define a homeomorphism $f: Y \rightarrow Y$ by

$$
f(y)= \begin{cases}\widetilde{g}(y) & \text { if } y \in(G, g), \\ \widetilde{g}^{-1}(y) & \text { if } y \in(G, g)^{\prime} .\end{cases}
$$

Then $f$ is an expansive homeomorphism and for both $\sigma=\mathrm{s}$ and $\mathrm{u}, W^{\sigma}(\widetilde{0})$ is not equal to the arc component $A(\widetilde{0})$.

Also, (3.10) is not true in the case that the shift map $\tilde{f}:(G, f) \rightarrow$ $(G, f)$ of $f$ is continuum-wise expansive, where $f: G \rightarrow G$ is a map of a graph $G$. In fact, there is a map $f: I \rightarrow I$ of the unit interval $I$ such that $f:(I, f) \rightarrow(I, f)$ is a continuum-wise expansive homeomorphism and $(I, f)$ is a pseudo-arc (= hereditarily indecomposable arc-like continuum) (see [16, $(2.3)])$. Since $(I, f)$ contains no arc and $W^{\mathrm{u}}(\widetilde{x})$ contains a nondegenerate subcontinuum of $(I, f)$ for each $\widetilde{x} \in(I, f)$ (see the proof of $[15,(3.2)]$ ), $W^{\mathrm{u}}(\widetilde{x})$ is not equal to the arc component $A(\widetilde{x})=\{\widetilde{x}\}$.

In connection with (3.10), we have the following questions.

QUESTION 1. In the situation of (3.10), under what assumptions does $X=(G, f)$ admit a closed neighborhood base $\left\{B_{n}\right\}_{n=1}^{\infty}$ such that each $B_{n}$ is the product of an arc in $W^{\mathrm{u}}$ and a Cantor set in $W^{\mathrm{s}}$ ? Is the condition that $X$ is $\sigma$-mixed sufficient? For Williams' mixing expanding maps on 1-dimensional branched manifolds, the answer is positive [27].

Question 2. Does "positive pseudo-expansiveness" imply "pseudoexpanding" in a metric giving the same topology as the original metric (cf. [3])?

The author wishes to thank the referee for his helpful and kind remarks.

\section{References}

[1] N. Aoki, Topological dynamics, in: Topics in General Topology, K. Morita and J. Nagata (eds.), Elsevier, 1989, 625-740.

[2] B. F. Bryant, Unstable self-homeomorphisms of a compact space, thesis, Vanderbilt University, 1954.

[3] M. Denker and M. Urba/nski, Absolutely continuous invariant measures for expansive rational maps with rationally indifferent periodic points, Forum Math. 3 (1991), 561-579.

[4] R. Devaney, An Introduction to Chaotic Dynamical Systems, 2nd ed., AddisonWesley, 1989.

[5] W. Gottschalk, Minimal sets; an introduction to topological dynamics, Bull. Amer. Math. Soc. 64 (1958), 336-351.

[6] W. Gottschalk and G. Hedlund, Topological Dynamics, Amer. Math. Soc. Colloq. Publ. 34, Amer. Math. Soc., 1955. 
[7] K. Hiraide, Expansive homeomorphisms on compact surfaces are pseudo-Anosov, Osaka J. Math. 27 (1990), 117-162.

[8] J. F. Jacobson and W. R. Utz, The nonexistence of expansive homeomorphisms of a closed 2-cell, Pacific J. Math. 10 (1960), 1319-1321.

[9] H. Kato, The nonexistence of expansive homeomorphisms of Peano continua in the plane, Topology Appl. 34 (1990), 161-165.

[10] - On expansiveness of shift homeomorphisms of inverse limits of graphs, Fund. Math. 137 (1991), 201-210.

[11] - , The nonexistence of expansive homeomorphisms of dendroids, ibid. 136 (1990), 37-43.

[12] - Embeddability into the plane and movability on inverse limits of graphs whose shift maps are expansive, Topology Appl. 43 (1992), 141-156.

[13] -, Expansive homeomorphisms in continuum theory, ibid. 45 (1992), 223-243.

[14] - Expansive homeomorphisms and indecomposability, Fund. Math. 139 (1991), 49-57.

[15] -, Continuum-wise expansive homeomorphisms, Canad. J. Math. 45 (1993), 576598.

[16] - Concerning continuum-wise fully expansive homeomorphisms of continua, Topology Appl., to appear.

[17] H. Kato and K. Kaw amura, A class of continua which admit no expansive homeomorphisms, Rocky Mountain J. Math. 22 (1992), 645-651.

[18] K. Kuratowski, Topology, Vol. II, Academic Press, New York, 1968.

[19] - Applications of Baire-category method to the problem of independent sets, Fund. Math. 81 (1974), 65-72.

[20] R. Mañé, Expansive homeomorphisms and topological dimension, Trans. Amer. Math. Soc. 252 (1979), 313-319.

[21] S. B. Nadler, Jr., Hyperspaces of Sets, Pure and Appl. Math. 49, Dekker, New York, 1978.

[22] R. V. Plykin, Sources and sinks of A-diffeomorphisms of surfaces, Math. USSRSb. 23 (1974), 233-253.

[23] - On the geometry of hyperbolic attractors of smooth cascades, Russian Math. Surveys 39 (1984), 85-131.

[24] W. Reddy, The existence of expansive homeomorphisms of manifolds, Duke Math. J. 32 (1965), 627-632.

[25] P. Walters, An Introduction to Ergodic Theory, Graduate Texts in Math. 79, Springer, 1982.

[26] R. F. Williams, A note on unstable homeomorphisms, Proc. Amer. Math. Soc. 6 (1955), 308-309.

[27] -, One-dimensional non-wandering sets, Topology 6 (1967), 473-487.

FACULTY OF INTEGRATED ARTS AND SCIENCES

HIROSHIMA UNIVERSITY

1-7-1 KAGAMIYAMA, HIGASHI-HIROSHIMA

724 JAPAN

Received 19 November 1992;

in revised form 15 March and 6 April 1993 